\title{
The Korean Society for Neuro-Oncology (KSNO) Guideline for Glioblastomas: Version 2018.01
}

\author{
Young Zoon Kim${ }^{1}$, Chae-Yong Kim², Jaejoon Lim³ ${ }^{3}$ Kyoung Su Sung ${ }^{4}$, Jihae Lee ${ }^{5}$, Hyuk-Jin Oh ${ }^{6}$, Seok-Gu Kang ${ }^{7}$, Shin-Hyuk Kang ${ }^{8}$, \\ Doo-Sik Kong ${ }^{9}$, Sung Hwan Kim ${ }^{10}$, Se-Hyuk Kim ${ }^{11}$, Se Hoon Kim² ${ }^{12}$, Yu Jung Kim ${ }^{13}$, Eui Hyun Kim², In Ah Kim ${ }^{14}$, Ho Sung Kim ${ }^{15}$, \\ Tae Hoon Roh ${ }^{11}$, Jae-Sung Park ${ }^{16}$, Hyun Jin Park ${ }^{17}$, Sang Woo Song ${ }^{18}$, Seung Ho Yang ${ }^{19}$, Wan-Soo Yoon ${ }^{20}$, Hong In Yoon ${ }^{21}$, \\ Soon-Tae Lee ${ }^{22}$, Sea-Won Lee ${ }^{23}$, Youn Soo Lee ${ }^{24}$, Chan Woo Wee ${ }^{14}$, Jong Hee Chang ${ }^{7}$, Tae-Young Jung ${ }^{25}$, Hye Lim Jung ${ }^{26}$, \\ Jae Ho Cho ${ }^{21}$, Seung Hong Choi ${ }^{27}$, Hyoung Soo Choi ${ }^{28}$, Je Beom Hong ${ }^{29}$, Do Hoon Lim ${ }^{30 *}$, Dong-Sup Chung ${ }^{20 *}$; \\ KSNO Guideline Working Group
}

\author{
Received February 25, 2019 \\ Revised March 19, 2019 \\ Accepted March 27, 2019 \\ Correspondence \\ Do Hoon Lim \\ Department of Radiation Oncology, \\ Samsung Medical Center, \\ Sungkyunkwan University \\ School of Medicine, 81 Irwon-ro, \\ Gangnam-gu, Seoul 06351, Korea \\ Tel: +82-2-3410-2600 \\ Fax: +82-3410-2609 \\ E-mail: dh8lim@skku.edu \\ Dong-Sup Chung \\ Department of Neurosurgery, \\ Incheon St. Mary's Hospital, \\ College of Medicine, The Catholic \\ University of Korea, 56 Dongsu-ro, \\ Bupyeong-gu, Incheon 21431, Korea \\ Tel: +82-32-280-5841 \\ Fax: +82-32-280-5991 \\ E-mail: dschung@catholic.ac.kr \\ *These authors contributed equally to \\ this work as a corresponding author.
}

Background There has been no practical guidelines for the management of patients with central nervous system (CNS) tumors in Korea for many years. Thus, the Korean Society for Neuro-Oncology (KSNO), a multidisciplinary academic society, started to prepare guidelines for CNS tumors from February 2018.

Methods The Working Group was composed of 35 multidisciplinary medical experts in Korea. References were identified through searches of PubMed, MEDLINE, EMBASE, and Cochrane CENTRAL using specific and sensitive keywords as well as combinations of keywords.

Results First, the maximal safe resection if feasible is recommended. After the diagnosis of a glioblastoma with neurosurgical intervention, patients aged $\leq 70$ years with good performance should be treated by concurrent chemoradiotherapy with temozolomide followed by adjuvant temozolomide chemotherapy (Stupp's protocol) or standard brain radiotherapy alone. However, those with poor performance should be treated by hypofractionated brain radiotherapy (preferred) \pm concurrent or adjuvant temozolomide, temozolomide alone (Level III), or supportive treatment. Alternatively, patients aged $>70$ years with good performance should be treated by hypofractionated brain radiotherapy+concurrent and adjuvant temozolomide or Stupp's protocol or hypofractionated brain radiotherapy alone, while those with poor performance should be treated by hypofractionated brain radiotherapy alone or temozolomide chemotherapy if the patient has methylated MGMT gene promoter (Level III), or supportive treatment.

Conclusion The KSNO's guideline recommends that glioblastomas should be treated by maximal safe resection, if feasible, followed by radiotherapy and/or chemotherapy according to the individual comprehensive condition of the patient.

Key Words Korean Society for Neuro-Oncology; Guideline; Glioblastomas; Practice.

\section{INTRODUCTION}

Glioblastoma is the most common malignant tumor of the central nervous system (CNS), accounting for approximately $12-15 \%$ of all primary intracranial neoplasms and $60-75 \%$ of glial tumors [1,2]. In most European and North American countries and Australia, the annual incidence is about 3-4 cases per 100,000 population [1]. Its incidence is relatively low in Korea, with 0.59 cases per 100,000 population per year [3]. In 2004, a randomized phase III trial with glioblastoma patients showed that concomitant chemoradiotherapy using temozolomide and adjuvant temozolomide in addition to standard postoperative radiotherapy improved the length of survival compared to postoperative radiotherapy alone [4].

This is an Open Access article distributed under the terms of the Creative Commons Attribution Non-Commercial License (https://creativecommons.org/licenses/by-nc/4.0) which permits unrestricted non-commercial use, distribution, and reproduction in any medium, provided the original work is properly cited.

Copyright ๑ 2019 The Korean Brain Tumor Society, The Korean Society for Neuro-Oncology, and The Korean Society for Pediatric Neuro-Oncology 
According to a long-term follow-up of a recent randomized phase III clinical trial and final results of the trial in 2009 [5], this therapeutic approach using temozolomide was considered the recent standard treatment for glioblastoma patients as it increased the median length of survival to $12-15$ months and showed better outcome for patients with methylated promoter of the O6-methyl guanine DNA methyltransferase (MGMT) gene.

To date, many clinical trials have been performed. Many trials are also ongoing to overcome this disastrous disease. Several new therapeutic modalities such as alternating electric field therapy [6] and immunotherapy agents [7] have been developed. To apply these new therapeutic strategies for glioblastoma patients, well established cancer networks or academic societies for CNS tumors of western countries such as the National Comprehensive Cancer Network (NCCN) $[8,9]$ and the European Association for Neuro-Oncology (EANO) [10] are updating their guidelines for CNS tumor regularly. Therapeutic strategies can be modified according to individual status of the patient known to be prognostic factors for survival in glioblastoma patients including the age of patient, performance status, surgical extent, recursive partitioning analysis class, methylation status of MGMT gene promoter, and postoperative chemotherapy and/or radiotherapy [5]. Therefore, a practical guideline is helpful for patients in detail.

However, the atmosphere for treating glioblastoma patients in Korea is different from that in other countries. In Korea, the incidence of glioblastoma is relatively lower [3] and the overall survival of patients seems to be longer [11] than that of western countries. Korean government supports medical care financially by the National Health Insurance System which has several limitations to apply updated therapeutic modalities to patients.

A practical guideline for the management of patients with CNS tumors in Korea is not available. Therefore, the Korean Society for Neuro-Oncology (KSNO), a multidisciplinary academic society for CNS tumors, bagan to prepare a guideline from February 2018. The objective of this guideline is to provide physicians with evidence-based recommendations and consensus expert opinion for the management of patients with glioblastomas in daily clinical practice. It will also serve as a source of knowledge for institutions and insurance companies involved in cancer care in Korea.

\section{KSNO GUIDELINE WORKING GROUP}

A Working Group was appointed by the KSNO in February 2018 to establish guidelines on the management of glioblastoma patients. The guidelines should be optimized considering the unique medical circumstance in Korea. The Working
Group was composed of 35 medical experts in Korea, including 18 neurosurgeons, 8 radiation oncologists, 1 medical oncologist, 2 neuroradiologists, 3 pediatric oncologists, 2 pathologists, and 1 neurologist. As there is no medical specialty for neuro-oncology in Korea, neurosurgeons (especially brain tumor surgeon) usually play a role of neuro-oncologists in clinical practice.

References were identified through searches of PubMed, MEDLINE, EMBASE, and Cochrane CENTRAL using specific and sensitive keywords as well as combinations of keywords. Abstracts presented at the annual meeting of KSNO in September 2018 and the official year-end conference of KSNO in December 2018 were also considered relevant. When available, we also collected existing guidelines from national multidisciplinary neuro-oncological societies such as the NCCN and EANO. The final reference list was generated based on originality and relevance to the scope of this guideline. The strategy of establishing this guideline was mainly based on NCCN and EANO guidelines with modifications and changes according to the unique background of Korea.

Scientific evidence was assessed and graded according to the following categories: high level evidence (evaluated from multiple populations and derived from randomized clinical trials or meta-analysis or systemic review), and low level evidence (evaluated from limited population and derived from non-randomized studies, including observational studies, cohort studies, and case-control studies).

To establish recommendation levels, the following criteria were used. Level I (strong recommendation) required a high level evidence and uniform consensus among panels. Level II (weak recommendation) required a high level evidence but not uniform consensus among panels or low level evidence but uniform consensus among panels. Level III (no consensus; individual decision) required a low level evidence but not uniform consensus among panels. Level IV (not recommendable) required contents being not beneficial or harmful. Recommendations with level I and level II evidence were not marked. However, those with level III and level IV evidence were marked at the end of each recommendation in this guideline.

\section{DIAGNOSIS OF GLIOBLASTOMAS}

When the radiological feature suggests a high-grade glioma, multidisciplinary approach for treatment planning should be considered if feasible (Fig. 1). Computed tomography (CT) is insufficient to suggest a high-grade glioma even with contrast enhancement. Therefore, magnetic resonance image (MRI) with contrast enhancement is essential to diagnose a high-grade glioma. In order to obtain sufficient tissue for his- 


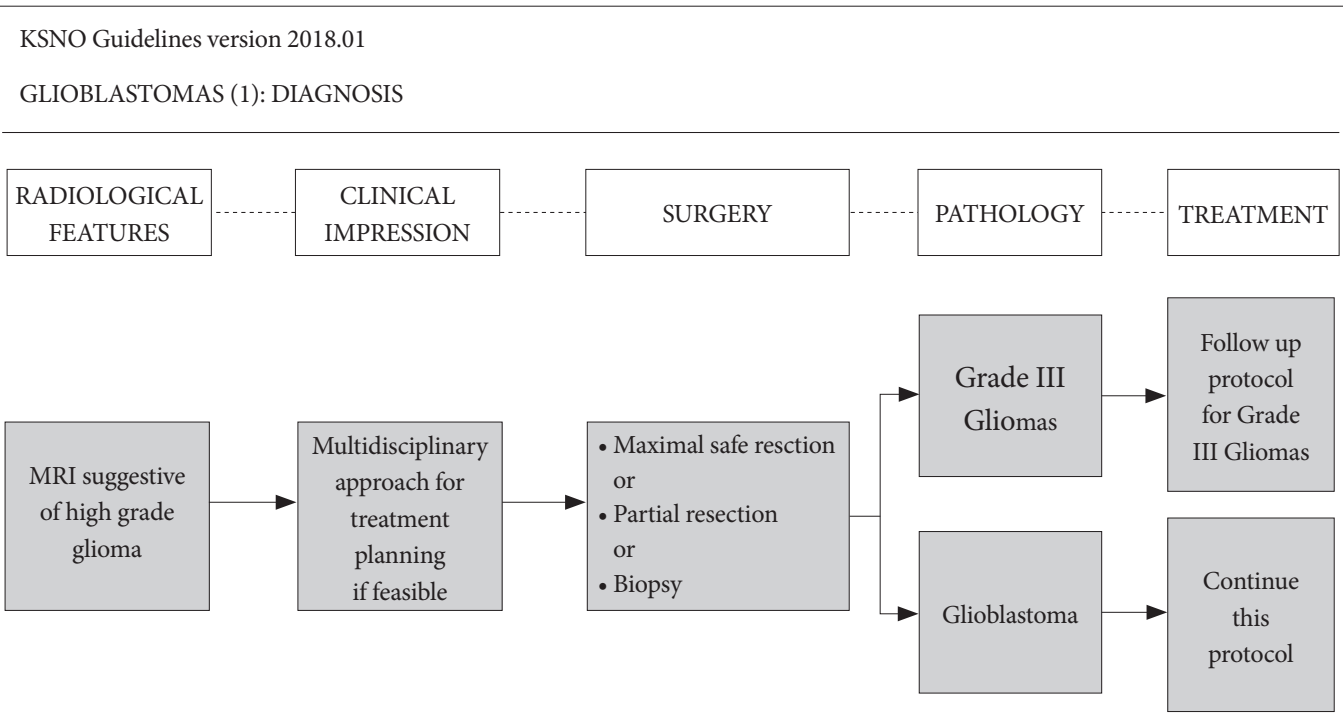

Fig. 1. Guideline for diagnosis of glioblastoma. To diagnose glioblastoma, MRI with gadolinium enhancement is essential. A multidisciplinary approach for treatment planning is recommended if feasible. Glioblastoma should be diagnosed histopathologically for tissues obtained by neurosurgical intervention. KSNO, Korean Society for Neuro-Oncology; MRI, magnetic resonance image.

topathological diagnosis, neurosurgical intervention is mandatory even if it is for stereotactic biopsy. To achieve maximal safe resection, neuronavigation systems, intraoperative CT or MRI, intraoperative ultrasonography, intraoperative mapping techniques, and fluorescence-guidance with 5-aminolevuliniv acid are recommended. Histopathological diagnosis should be officially based on World Health Organization (WHO) 2016 Classification of Tumors of the Central Nervous System [12]. Codeletion of $1 \mathrm{p} / 19 \mathrm{q}$ testing and MGMT promoter methylation test are essential parts of molecular diagnostics for glioblastomas. Isocitrate dehydrogenase 1 and 2 (IDH1 and IDH2) mutation test is also required for workup of glioblastomas.

\section{ADJUVANT TREATMENT OF GLIOBLASTOMAS}

Among glioblastoma patients aged $\leq 70$ years, if the patient has good performance status [Karnofsky Performance Scale (KPS) $\geq 60$ or Eastern Cooperative Oncology Group (ECOG) performance score $\leq 2$ ], concurrent chemoradiotherapy with temozolomide followed by adjuvant temozolomide chemotherapy (Stupp's protocol) [13] or standard brain radiotherapy alone should be considered. If the patient has poor performance status (KPS $<60$ or ECOG performance score $\geq 3$ ), hypofractionated brain radiotherapy (preferred) \pm concurrent or adjuvant temozolomide, temozolomide alone (Level III), or supportive treatment can be considered. Optional treatment of temozolomide alone for younger glioblastoma patients with poor performance had low level of evidence without uniform consensus but was recommended. It was decided to be included in this guideline (Fig. 2).

On the contrary, for glioblastoma patients aged $>70$ years, if the patient has good performance status (KPS $\geq 60$ or ECOG performance score $\leq 2$ ), the following therapeutic options should be considered: hypofractionated brain radiotherapy+ concurrent and adjuvant temozolomide, or concurrent chemoradiotherapy with temozolomide followed by adjuvant temozolomide chemotherapy, or hypofractionated brain radiotherapy alone. If the patient has poor performance status (KPS $<60$ or ECOG performance score $\geq 3$ ), the following therapeutic options should be considered: hypofractionated brain radiotherapy alone, temozolomide chemotherapy if methylated MGMT gene promoter (Level III) is present, or supportive treatment. In poorly performing patients or the elderly, a hypofractionated accelerated course is reasonable with the goal of completing the treatment in 2-3 weeks. Optional treatment of temozolomide alone for older glioblastoma patients with poor performance also had low level evidence without uniform consensus but was recommended if he/she had a methylated MGMT gene promoter. It was decided to be included in this guideline (Fig. 3).

Radiological follow-up using MRI with gadolinium enhancement is recommended for regular check-up 2-6 weeks after radiotherapy, then every 2-4 months for 3 years, then every 6 months regardless of age or therapeutic options (Fig. $2,3)$. There is a uniform consensus among the panel for routine check of MRI at 2-6 weeks after radiotherapy. In fact, MRI at this time can be very informative to detect radiationinduced brain swelling and pseudoprogression after radiotherapy. 


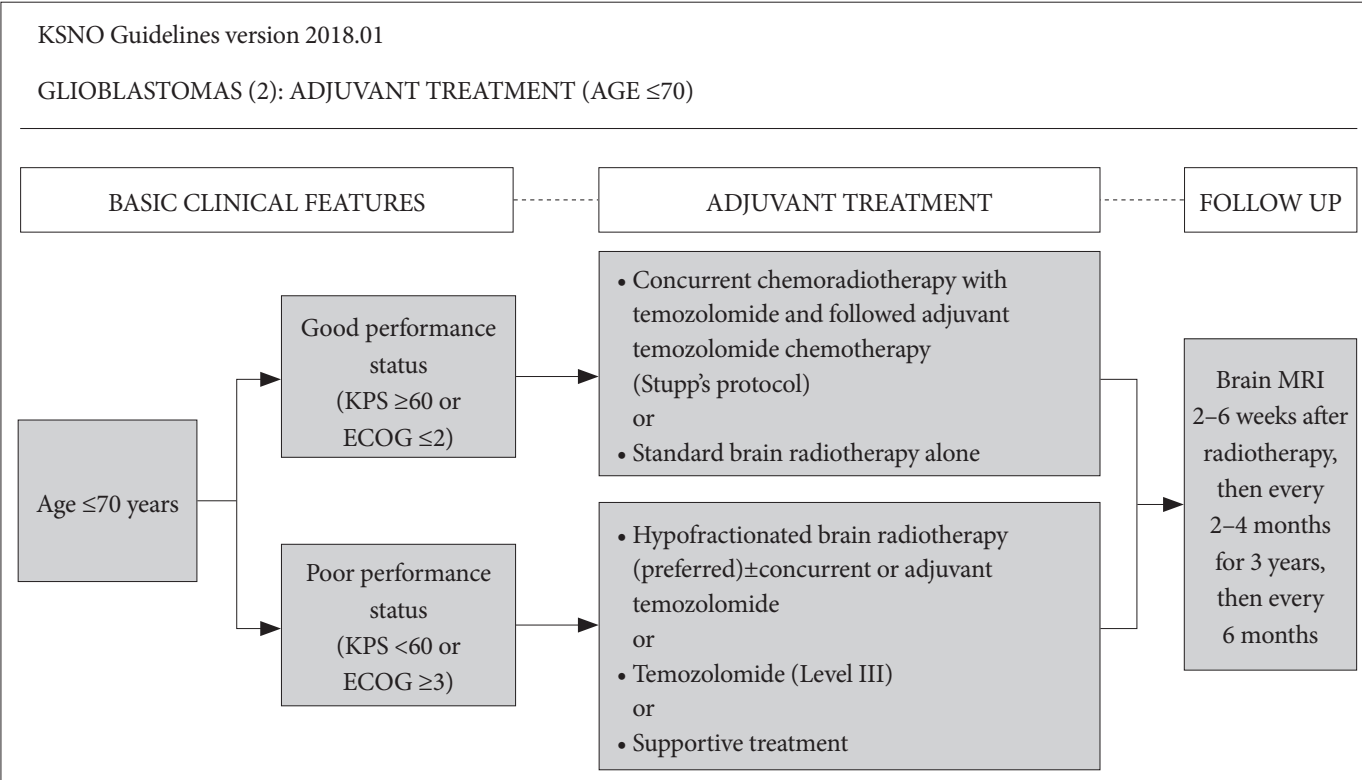

Fig. 2. Guideline for patients with age $\leq 70$ years. Patients with good performance are recommended to be treated by concurrent chemoradiotherapy with temozolomide followed by adjuvant temozolomide chemotherapy (Stupp's protocol) or standard brain radiotherapy alone. However, those with poor performance are recommended to be treated by hypofractionated brain radiotherapy (preferred) \pm concurrent or adjuvant temozolomide, temozolomide alone (Level III), or supportive treatment. KSNO, Korean Society for Neuro-Oncology; KPS, Karnofsky Performance Scale; ECOG, Eastern Cooperative Oncology Group; MRI, magnetic resonance image.

KSNO Guidelines version 2018.01

GLIOBLASTOMAS (3): ADJUVANT TREATMENT (AGE >70)

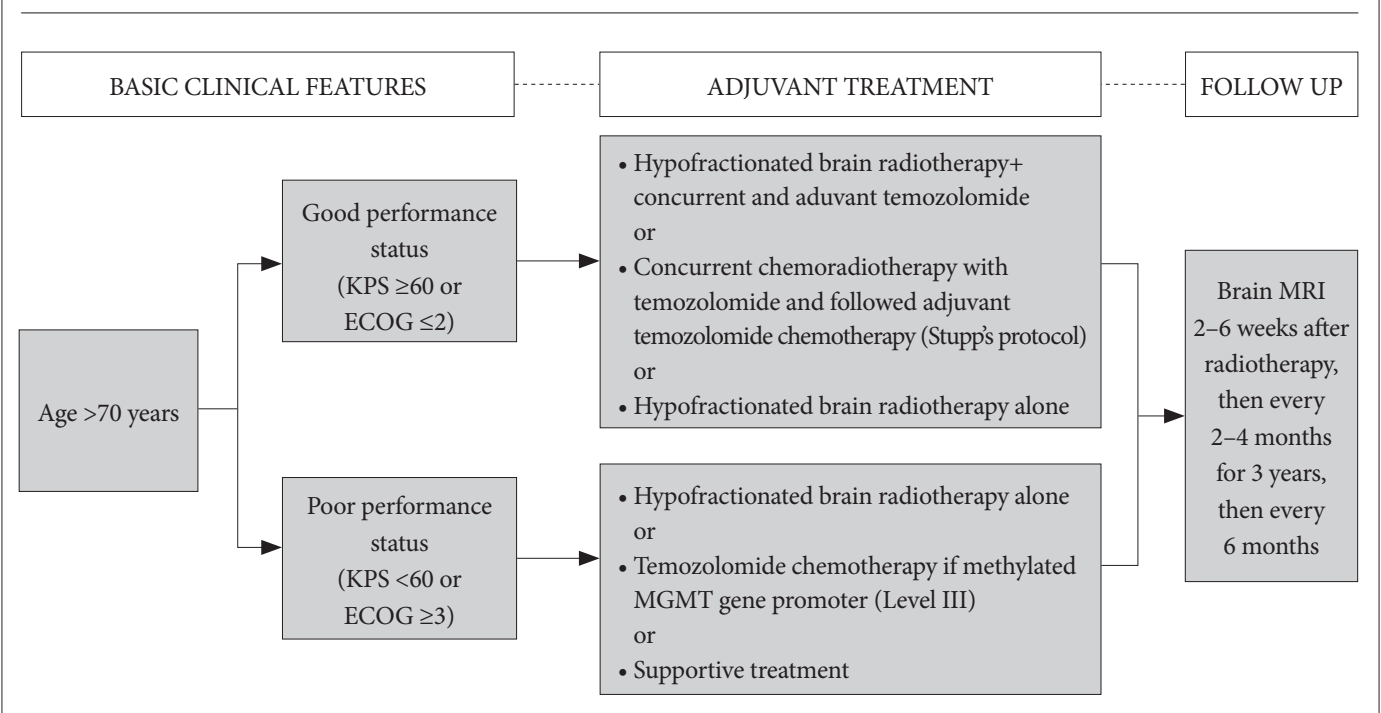

Fig. 3. Guideline for patients with age $>70$ years. Patients with good performance are recommended to be treated by hypofractionated brain radiotherapy+concurrent and adjuvant temozolomide, Stupp's protocol, or hypofractionated brain radiotherapy alone. However, those with poor performance are recommended to be treated by hypofractionated brain radiotherapy alone, temozolomide chemotherapy alone if they have a methylated MGMT gene promoter (Level III), or supportive treatment. KSNO, Korean Society for Neuro-Oncology; KPS, Karnofsky Performance Scale; ECOG, Eastern Cooperative Oncology Group; MRI, magnetic resonance image.

\section{TREATMENT OF RECURRENT GLIOBLASTOMA}

Diagnosis of glioblastoma recurrence can be indistinguish- able from pseudoprogression on MRI within the first 3 months after completion of concurrent chemoradiotherapy with temozolomide. But, the following radiologic findings can suggest recurrence of glioblastomas; 1) $25 \%$ or more increase in 
enhancing lesions despite stable or increasing steroid dose, 2) significant increase of the lesion in the fluid-attenuated inversion recovery (FLAIR) image and T2 weighted image, not attributable to other non-tumor causes, and 3) any new lesions. Also, if clinical deterioration (not attributable to other non-tumor causes and not due to steroid decrease) occurs simultaneously, true progression is strongly suggested. If recurrence is suspected in conventional MRI, it is better to consider the following options; 1) undergoing biopsy, 2) checking functional radiologic study such as MR spectroscopy, MR perfusion, brain positron emission tomography (PET)-CT, or 3) checking MRI again and comparing changes that may be due to progression versus radiation necrosis. When glioblastoma recurrence is suggested clinically and radiologically, surgical resection is always recommended, if feasible (Fig. 4). Even for surgically unresectable diseases such as diffuse, multifocal, and deep located lesions, surgical treatment can be considered to reduce the mass effect and improve neurological symptoms. After surgical resection, the following therapeutic options are considered: systemic chemotherapy [bevacizumab alone, bevacizumab+irinotecan, daily temozolomide with low dose, lomustine or carmustine, PCV (procarbazine+carmustine +vincristine) or procarbazine+carmustine], and/or reirradiation (especially if long interval since prior to radiotherapy and/ or if there was a good response to prior radiotherapy), and/or supportive treatment if the patient has poor performance status (Fig. 4). The efficacy of standard-of-care treatment such as adjuvant temozolomide chemotherapy for recurrent glioblastoma is suboptimal for salvage purpose. Thus, for eligible patients, consideration of clinical trials is highly encouraged.

\section{PRINCIPLES OF THE MANAGEMENT OF GLIOBLASTOMAS}

\section{Brain imaging}

Many imaging modalities are available and used in neurooncology primarily to make treatment decisions in Korea. Imaging is always recommended to investigate emergent signs or symptoms.

MRI of the brain (with and without contrast) is the gold standard modality to investigate brain tumors. It provides a static picture of brain tumors. It has a benefit of providing a reasonably good delineation of tumors. In MRI, high grade tumors and brain leptomeningeal metastases usually show enhancement while low-grade tumors usually do not. However, it has a limitation in that it is sensitive to movement. In addition, metallic objects can cause artifacts. Thus, patients with implantable devices cannot receive an MRI. Moreover, claustrophobia or renal insufficiency may be an issue. Postoperative brain MRI should be performed within 24-72 hours after surgery for gliomas and other tumors to determine the extent of resection.

CT scans of the brain (with and without contrast) is usually considered for patients who cannot undergo an MRI. CT

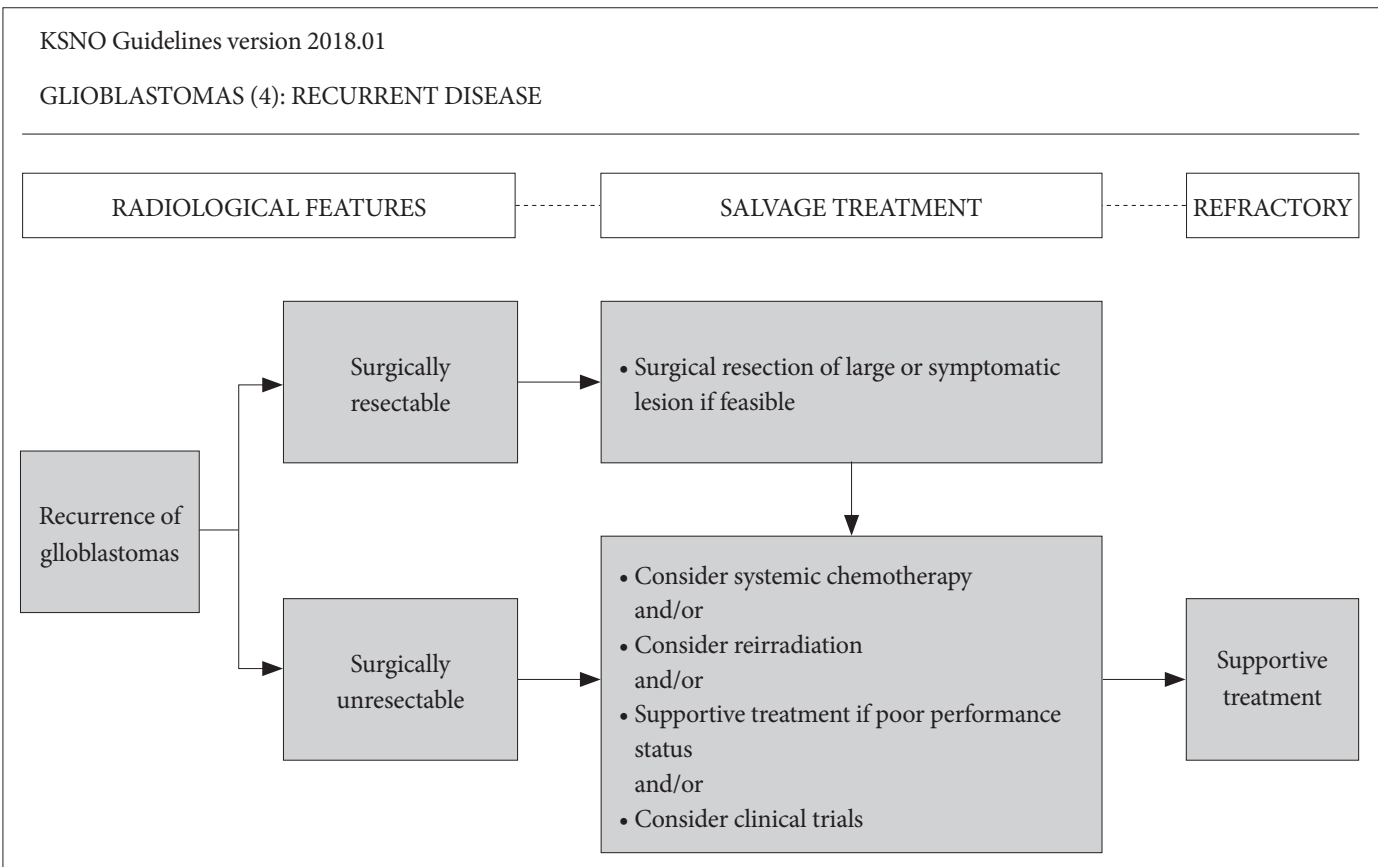

Fig. 4. Guideline for recurrent glioblastoma. Surgical resection is always recommended if feasible. Even in surgically unresectable diseases such as diffuse, multi-focal, and deep located lesions, surgical treatment can be considered to reduce the mass effect and improve neurological symptoms. After surgical resection, the following therapeutic options are considered: systemic chemotherapy, and/or reirradiation, and/or supportive treatment if poor performance status, and/or enrollment of clinical trials. KSNO, Korean Society for Neuro-Oncology. 
has a benefit of avoiding claustrophobia or implanted devices in the body. It is faster than an MRI. However, CT has a limitation in that it lacks resolution, especially in a tumor located within the posterior fossa, and for the patients with renal insufficiency.

MR spectroscopy can be used to assess metabolites within tumor and normal tissues. It may be useful for differentiating tumors from radiation necrosis. It may also be helpful in grading tumors or assessing therapeutic response. The area showing the most abnormal features would be the best place to target for a biopsy. However, it has limitation for tumors near vessels, air space, or bone.

MR perfusion can be used to measure cerebral blood volume in tumors. It may be useful for differentiating the grade of tumor or tumor versus radiation necrosis. The area with the highest perfusion would be the best place for a biopsy. However, it has limitation for tumors near vessels, air space, and bone, or small-volume lesions.

PET-CT using fludeoxyglucose (FDG) has a limitation for functional diagnosis of glioblastomas due to high uptake of FDG in the brain with normal biologic metabolism. However, PET-CT using methionine, which is an essential amino acid, may be helpful for differentiating the grade of tumor or tumor versus radiation necrosis. But this technology is not commonly used in the clinical field in Korea, further development of application is necessary for casual use in the clinical practice.

\section{Multidisciplinary care}

During treatment, most glioblastoma patients will be managed by various subspecialists. Close and regular communication among all providers across multidisciplines is essential. Utilization of a brain tumor board or multidisciplinary clinic care models can facilitate interactions among various subspecialists, ideally including allied health services (e.g., physical, occupational and speech therapies, nursing, psychology, and social services) to optimize treatment plan recommendations.

As treatment proceeds, it is important that the patient and his/her family understand the role of each team member. One attending physician who mainly cares for the patient should be determined as early as possible and the attending physician should contact the patients regularly for follow up. Additionally the attending physician can facilitate referral to the appropriate specialist.

The patient is strongly encouraged to participate in various clinical trials. Practitioners should discuss any local, regional, and national options for which the patient may be eligible and advantages and disadvantages of participation. The center that treats neuro-oncology patients should encourage patients to participate in large collaborative trials in order to have another option to offer patients.
Throughout treatment, patient's health-related quality of life should remain the highest priority and guide clinical decision-making. While therapeutic response on the radiologic study can be a good indicator of successful therapy, other nonradiologic indicators of therapeutic response such as overall well-being, cognitive function, physical and motor functioning in day-to-day activities, communication ability, social functioning and family interactions, nutrition, pain control, longterm consequences of treatment, and psychological issues must also be considered.

Patients should be informed of the possibility of pseudoprogression, its approximate incidence, and potential investigations that may be needed if pseudoprogression is suspected. Close follow-up imaging, MR spectroscopy, PET-CT imaging, and repeat neurosurgical intervention including biopsy or surgical resection may be necessary, if clinically indicated.

\section{Brain tumor surgery}

General principles of surgical resection of glioblastoma are as follows: gross total resection when appropriate, minimal surgical morbidity, and accurate diagnosis. The following factors should be considered when deciding the surgical resection: age, performance status, feasibility of decreasing the mass effect with surgery, resectability, including number of lesions, location of lesions, time since last surgery in recurrent patients, and new versus recurrent tumor [9].

Options of surgical resection include gross total resection where feasible, stereotactic biopsy, and open biopsy/debulking followed by planned observation or adjuvant therapy. In order to obtain the maximal safe resection, neuronavigation systems, intraoperative MRI or CT, intraoperative ultrasonography, fluorescence-guided with 5 -aminolevulinic acid, and intraoperative mapping techniques may be helpful.

For histopathological diagnosis and genetic information, sufficient tissue should be sent to the pathologist for neuropathology evaluation and molecular correlates. Frozen section analysis, when possible, can help with intraoperative decision making. The tissue should be reviewed by an experienced neuropathologist.

Postoperative brain MRI should be performed within 2472 hours after surgery for glioblastomas to determine the extent of resection. The extent of resection should be judged based on postoperative imaging study and used as a baseline to assess further therapeutic efficacy or tumor progression.

\section{Pathology examination}

Incorporation of relevant diagnostic markers, including histopathologic and molecular information, which was described at the WHO 2016 Classification of Tumors of the Central Nervous System, should be considered as standard practice for 
tumor classification. Molecular/genetic characterization complements standard histologic analysis, providing additional diagnostic and prognostic information that can greatly improve diagnostic accuracy, influence treatment selection, and possibly improve management decision-making.

For standard histopathologic examination of glioblastomas, basic histologic examination is performed based on the description in the WHO Classification of Tumors of the Central Nervous System [12]. Inter-observer discrepancies in histologic diagnosis and grading are recognized issues due to the inherently subjective nature of certain aspects of histopathologic interpretation (e.g., astrocytic vs. oligodendroglial morphology). In addition, surgical sampling does not always capture all relevant diagnostic features in morphologically heterogeneous tumors.

Through genetic and molecular testing, glioblastomas can be differentiated more accurately in terms of prognosis and, in some instances, for response to different therapies. However, molecular/genetic characterization does not replace standard histologic assessment. It serves as a complementary approach to provide additional diagnostic and prognostic information that often enhances treatment selection. Although there are no identified targeted agents with demonstrate efficacy in glioblastoma, the panel encourages molecular testing of tumors such as next generation sequencing because if a driver mutation is detected, it may be reasonable to treat patient with a targeted therapy on a compassionate use basis, and/ or the patient may have more treatment options in the context of a clinical trial. Molecular testing also has a valuable role in improving diagnostic accuracy and prognostic stratification that may inform treatment selection. For example, MGMT promoter methylation is an essential part of molecular diagnostics for all high-grade gliomas and $1 \mathrm{p} / 19 \mathrm{q}$ codeletion testing is an essential part of molecular diagnostics for oligodendrogliomas. Therefore, $1 \mathrm{p} / 19 \mathrm{q}$ codeletion testing is considered to differentiate astrocytoma from oligodendrogliomas. Also, $I D H 1$ and $I D H 2$ mutation testing is required for differential diagnosis between $I D H$ mutant and $I D H$ wildtype tumors.

\section{Radiotherapy}

Optimal timing of radiotherapy after surgical resection is not established in glioblastomas. Delay in radiotherapy has not been shown to decrease survival. However, we recommend radiotherapy to be initiated at postoperative 2-6 weeks after full recovery from surgical interventions. Whenever radiotherapy is planned for glioblastoma patients, pre- and postoperative MRIs should be performed to define all target volumes, including gross and clinical tumor volume (GTV and CTV). Additional MRI at the time of radiotherapy simu- lation can be used to account for changes in surgical cavity or lesions. CT-based 3-dimensional calculation of dose distribution should be performed at any circumstance.

The GTV should encompass preoperative tumor bed and enhancing lesions on T1-weighted image of postoperative MRI. The preoperative tumor bed should not be directly delineated on the registered preoperative MRI on the planning $\mathrm{CT}$ in patients undergoing surgical resection. T2/FLAIR signals can also be included in the GTV if needed. Historical trials and international groups (e.g., RTOG and EORTC) generally recommend an expansion of 2-2.5 cm for CTV delineation to account for subclinical tumor infiltration. However, smaller CTV-margins may be reasonable as supported by retrospective studies (as low as $0.5 \mathrm{~cm}$ ). Simple expansion from the GTV should be avoided. CTV should always be modified based on anatomical barriers for tumor infiltrations. A margin of 3-5 mm from the CTV is usually recommended to create the planning target volume to account for errors from image-registration and daily set-up of patients. However, the margin can also be reduced if daily image-guidance is performed.

A total dose of 59.4-61.2 Gy is recommended as standard radiotherapy using a daily fraction of 1.8-2.0 Gy. A reduced field can be used after delivering 45-50.4 Gy. However, field reduction does not always need to be performed sequentially. When intensity-modulated radiotherapy (IMRT or IMPT) is used to avoid critical organs, simultaneous integrated boost techniques can be used. For fragile patients with old age or poor performance requiring hypofractionated radiotherapy in 1-4 weeks, the following dose-fractionation regimens are frequently adopted: $40.05 \mathrm{~Gy} / 15$ fractions, $34 \mathrm{~Gy} / 10$ fractions, $50 \mathrm{~Gy} / 20$ fractions, and $25 \mathrm{~Gy} / 5$ fractions. No single scheme has been proven to be superior to another. Different schedules can also be used based on physician's decision. The optimal margin for CTV expansion has not been established yet. Therefore, we recommend physicians to follow the principles of standard radiotherapy. Absolute cumulative dose limits and optimal interval between radiotherapy sessions are not established for glioblastomas. Delivery, dose, fraction, target volume, and techniques for reirradiation should be decided by a brain tumor radiation oncologist and through a multidisciplinary discussion whenever feasible.

\section{DISCUSSION}

We differentiated adjuvant therapeutic strategies based on the age of 70 in this study. However, there is no concrete definition of old age. Many investigators have reported their data based on old age of $>70$ years old. Although there was uniform consensus for old age of $>70$ among panels, a nation-wide retrospective multi-institute study can help establish the mean- 
ingful age that has the prognostic turning point in Korea.

As mentioned above, almost all medical practices are under regulation by the National Health Insurance System in Korea. Limited therapeutic modalities were included in this guideline. This is because the national system does not cover various therapeutic modalities such as electric field therapy, thus limiting clinical practice in the field of neuro-oncology in Korea. The Korean government permits only two therapeutic options such as concurrent chemoradiotherapy with temozolomide and standard brain radiotherapy alone regardless of the methylation status of MGMT promoter after surgery. Therefore, this guideline does not divide the adjuvant treatment according to MGMT promoter methylation status as in other guidelines. Although the Korean National Health Insurance System does not permit single adjuvant treatment using temozolomide after surgery of glioblastoma, the panel has a consensus that this therapeutic option can be considered for patients with poor performance status, even in young age, with $150-200 \mathrm{mg} / \mathrm{m}^{2}$ for $5 / 28$ schedule.

In terms of molecular and genetic diagnosis, only three tests were included in this guideline: methylation status of MGMT gene promoter, IDH 1/2 mutation, and $1 \mathrm{p} / 19 \mathrm{q}$ codeletion. The NCCN [9] and EANO guideline [10] recommend more tests for genetic alteration of glioblastomas, including ATRX mutation, TERT mutation, and H3F3A mutation. ATRX mutations in gliomas are strongly associated with $I D H$ mutations. They are nearly always mutually exclusive with $1 \mathrm{p} / 19 \mathrm{q}$ codeletion [14]. TERT mutations are almost invariably present in $1 \mathrm{p} / 19 \mathrm{q}$ codeleted oligodendrogliomas. They are found in most glioblastomas. TERT mutation, in combination with IDH mutation and $1 \mathrm{p} / 19 \mathrm{q}$ codeletion, is characteristic of oligodendrogliomas. Absence of TERT mutation, coupled with IDH mutation, designates an astrocytoma $[15,16]$. Histone mutations most commonly occur in pediatric midline gliomas (e.g., diffuse intrinsic pontine gliomas), although midline gliomas in adults can also contain histone mutations [17]. Their presence can be considered solid evidence of an infiltrative glioma. This is often helpful in small biopsies of midline lesions that may not be fully diagnostic with light microscopy or which do not fully resemble infiltrative gliomas [17]. However, these more complex and detail molecular tests were not included in this guideline due to practical limitations at individual institute in Korea.

At recurrence, there is no standard or effective treatment for glioblastomas in Korea or western countries. Much less regimens can be used for patients, including several alkylating agents in Korea, than those in other countries. Due to a smaller number of glioblastoma patients in Korea, global pharmaceutical companies are reluctant to enroll Korean patients into their clinical trials for new drugs such as immunotherapeutic agents. Therefore, chances to participate in clini- cal trials in Korea are very small. This background results in clinical trials to be recommended only at the time of recurrence in this guideline.

This guideline has a marked weakness of limited application to Korean patients with glioblastomas due to the unique medical atmosphere of Korea. Therefore, it is less helpful for physicians treating glioblastoma patients outside of Korea. In order to use this guideline globally, neighbor countries close to Korea such as Japan or China should have a comprehensive network for brain tumor management and cooperate and share their guidelines. Further, it will be helpful for Far Eastern countries to establish a global guideline that is commonly applicable to these countries. The next hurdle to be overcome is molecular and genetic test for glioblastomas and whole brain tumors. Genetic information is now rapidly changing, making it difficult to establish definite guideline for clinical practice. The Consortium to Inform Molecular and Practical Approaches to CNS Tumor Taxonomy (cIMPACT-NOW) is the notable example that shows rapid change in the molecular diagnosis of brain tumors $[18,19]$. Therefore, the KSNO's Guideline Working Group has plans to continue the process of updating guidelines so that the weakness of this version can be improved.

\section{CONCLUSIONS}

As there was no practical guideline for the management of brain tumor patients, the KSNO developed this guideline which should be applicable for physicians under unique medical circumstances in Korea. "The KSNO guideline for Glioblastoma: version 2018.01" is the first guideline prepared by the KSNO Guideline Working Group composed of 35 multidisciplinary medical experts in Korea. In summary, glioblastomas should be treated by maximal safe resection if feasible followed by radiotherapy and/or chemotherapy according to the individual comprehensive condition of patient. As data emerging in the past few years have led to significant changes in the diagnosis, categorization, and treatment of glioblasto$\mathrm{ma}$, we plan to update this guideline consistently. Also, consecutive guideline for other brain tumors such as gliomas, brain metastasis, and meningiomas will be published by the KSNO Guideline Working Group.

\section{Conflicts of Interest}

The authors have no potential conflicts of interest.

\section{Acknowledgments}

This protocol can be applied for the patients with WHO Grade IV Glioblastoma of the brain. We specially appreciate the following individuals for their help: Chan Woo Wee, M.D. (Department of Radiation Oncology, Seoul National University Bundang Hospital) for description of the principle of radiotherapy, Soon-Tae Lee, M.D. (Department of Neurology, Seoul 
National University Hospital) for description of the principle of multidisciplinary care, Jaejoon Lim, M.D. (Department of Neurosurgery, Bundang CHA Medical Center) for systemic review of chemotherapy for glioblastoma, Kyoung Su Sung, M.D. (Department of Neurosurgery, Dong-A University Hospital) for systemic review of surgical treatment for glioblastoma, Jihae Lee, M.D. (Department of Radiation Oncology, Ewha Women's University Mokdong Hospital) for systemic review of radiation therapy for glioblastoma, and Hyuk-Jin Oh, M.D. (Department of Neurosurgery, Soonchunhyang University Cheonan Hospital) for systemic review of treatment for recurrent glioblastoma.

\section{Author Affiliations}

${ }^{1}$ Division of Neurooncology and Department of Neurosurgery, Samsung Changwon Hospital, Sungkyunkwan University School of Medicine, Changwon, Korea; ${ }^{2}$ Department of Neurosurgery, Seoul National University Bundang Hospital, Seoul National University College of Medicine, Seongnam, Korea; ${ }^{3}$ Department of Neurosurgery, Bundang CHA Medical Center, CHA University, Seongnam, Korea; ${ }^{4}$ Department of Neurosurgery, Dong-A University Hospital, Dong-A University College of Medicine, Busan, Korea; ${ }^{5} \mathrm{De}-$ partment of Radiation Oncology, Ewha Women's University Mokdong Hospital, Ewha Women's University School of Medicine, Seoul, Korea; ${ }^{6}$ Department of Neurosurgery, Soonchunhyang University Cheonan Hospital, Soonchunhyang University College of Medicine, Cheonan, Korea; ${ }^{7}$ Department of Neurosurgery, Severance Hospital, Yonsei University College of Medicine, Seoul, Korea; ${ }^{8}$ Department of Neurosurgery, Korea University Anam Hospital, Korea University College of Medicine, Seoul, Korea; ${ }^{9}$ Department of Neurosurgery, Samsung Medical Center, Sungkyunkwan University School of Medicine, Seoul, Korea; ${ }^{10}$ Department of Radiation Oncology, St. Vincent's Hospital, College of Medicine, The Catholic University of Korea, Seoul, Korea; ${ }^{11}$ Department of Neurosurgery, Ajou University Hospital, Ajou University School of Medicine, Suwon, Korea; ${ }^{12}$ Department of Pathology, Severance Hospital, Yonsei University College of Medicine, Seoul, Korea; ${ }^{13} \mathrm{Di}$ vision of Medical Oncology, Department of Internal Medicine, Seoul National University Bundang Hospital, Seoul National University College of Medicine, Seongnam, Korea; ${ }^{14}$ Department of Radiation Oncology, Seoul National University Bundang Hospital, Seoul National University College of Medicine, Seongnam, Korea; ${ }^{15}$ Department of Radiology and Research Institute of Radiology, University of Ulsan College of Medicine, Asan Medical Center, Seoul, Korea; ${ }^{16}$ Department of Neurosurgery, Seoul St. Mary's Hospital, College of Medicine, The Catholic University of Korea, Seoul, Korea; ${ }^{17} \mathrm{Clin}-$ ic of Pediatric Oncology, National Cancer Center, Goyang, Korea; ${ }^{18}$ Department of Neurosurgery, Konkuk University Medical Center, Konkuk University School of Medicine, Seoul, Korea; ${ }^{19}$ Department of Neurosurgery, St. Vincent's Hospital, College of Medicine, The Catholic University of Korea, Seoul, Korea; ${ }^{20}$ Department of Neurosurgery, Incheon St. Mary's Hospital, College of Medicine, The Catholic University of Korea, Incheon, Korea; ${ }^{21}$ Department of Radiation Oncology, Yonsei Cancer Center, Yonsei University College of Medicine, Seoul, Korea; ${ }^{22}$ Department of Neurology, Seoul National University Hospital, Seoul National University College of Medicine, Seoul, Korea; ${ }^{23}$ Department of Radiation Oncology, Seoul St. Mary's Hospital, College of Medicine, The Catholic University of Korea, Seoul, Korea; ${ }^{24}$ Department of Pathology, Seoul St. Marry's Hospital, College of Medicine, The Catholic University of Korea, Seoul, Korea: ${ }^{25}$ Department of Neurosurgery, Chonnam National University Hwasun Hospital, Chonnam National University Medical School, Gwangju, Korea; ${ }^{26}$ Department of Pediatrics, Kangbuk Samsung Hospital, Sungkyunkwan University School of Medicine, Seoul, Korea; ${ }^{27}$ Department of Radiology, Seoul National University Hospital, Seoul National University College of Medicine, Seoul, Korea; ${ }^{28} \mathrm{De}-$ partment of Pediatrics, Seoul National University Bundang Hospital, Seoul National University College of Medicine, Seongnam, Korea; ${ }^{29}$ Department of Neurosurgery, Kangbuk Samsung Hospital, Sungkyunkwan University School of Medicine, Seoul, Korea; ${ }^{30}$ Department of Radiation Oncology, Samsung Medical Center, Sungkyunkwan University School of Medicine, Seoul, Korea

\section{REFERENCES}

1. Ostrom QT, Gittleman H, Truitt G, et al. CBTRUS statistical report: primary brain and central nervous system tumors diagnosed in the United States in 2011-2015. Neuro Oncol 2018;20(Suppl 4):iv1-86.

2. Louis DN, Ohgaki H, Wiestler OD, Cavenee WK, Ellison DW, Figarella-Branger D. Glioblastoma, IDH-wild-types. In: Louise DN, Brate DJ, Ohgaki H, et al., editors. WHO Classification of Tumours of the Central Nervous System. Rev 4th ed. Lyon: IARC Press; 2016. p. 28-45.

3. Dho YS, Jung KW, Ha J, et al. An updated nationwide epidemiology of primary brain tumors in Republic of Korea, 2013. Brain Tumor Res Treat 2017;5:16-23.

4. Hegi ME, Diserens AC, Gorlia T, et al. MGMT gene silencing and benefit from temozolomide in glioblastoma. N Engl J Med 2005;352:9971003.

5. Stupp R, Hegi ME, Mason WP, et al. Effects of radiotherapy with concomitant and adjuvant temozolomide versus radiotherapy alone on survival in glioblastoma in a randomised phase III study: 5-year analysis of the EORTC-NCIC trial. Lancet Oncol 2009;10:459-66.

6. Stupp R, Wong ET, Kanner AA, et al. NovoTTF-100A versus physician's choice chemotherapy in recurrent glioblastoma: a randomised phase III trial of a novel treatment modality. Eur J Cancer 2012;48:2192-202.

7. Maxwell R, Jackson CM, Lim M. Clinical trials investigating immune checkpoint blockade in glioblastoma. Curr Treat Options Oncol 2017; 18:51.

8. Nabors LB, Portnow J, Ammirati M, et al. Central nervous system cancers, version 1.2015. J Natl Compr Canc Netw 2015;13:1191-202.

9. Nabors LB, Portnow J, Ammirati M, et al. NCCN guidelines insights: central nervous system cancers, version 1.2017. J Natl Compr Canc Netw 2017;15:1331-45.

10. Weller M, van den Bent M, Hopkins K, et al. EANO guideline for the diagnosis and treatment of anaplastic gliomas and glioblastoma. Lancet Oncol 2014;15:e395-403.

11. Roh TH, Park HH, Kang SG, et al. Long-term outcomes of concomitant chemoradiotherapy with temozolomide for newly diagnosed glioblastoma patients: a single-center analysis. Medicine (Baltimore) 2017;96: e7422.

12. Louis DN, Perry A, Reifenberger G, et al. The 2016 World Health Organization classification of tumors of the central nervous system: a summary. Acta Neuropathol 2016;131:803-20.

13. Stupp R, Mason WP, van den Bent MJ, et al. Radiotherapy plus concomitant and adjuvant temozolomide for glioblastoma. N Engl J Med 2005; 352:987-96

14. Reuss DE, Sahm F, Schrimpf D, et al. ATRX and IDH1-R132H immunohistochemistry with subsequent copy number analysis and IDH sequencing as a basis for an "integrated" diagnostic approach for adult astrocytoma, oligodendroglioma and glioblastoma. Acta Neuropathol 2015;129:133-46.

15. Killela PJ, Reitman ZJ, Jiao Y, et al. TERT promoter mutations occur frequently in gliomas and a subset of tumors derived from cells with low rates of self-renewal. Proc Natl Acad Sci U S A 2013;110:6021-6.

16. Arita H, Yamasaki K, Matsushita Y, et al. A combination of TERT promoter mutation and MGMT methylation status predicts clinically relevant subgroups of newly diagnosed glioblastomas. Acta Neuropathol Commun 2016;4:79.

17. Meyronet D, Esteban-Mader M, Bonnet C, et al. Characteristics of H3 K27M-mutant gliomas in adults. Neuro Oncol 2017;19:1127-34.

18. Louis DN, Aldape K, Brat DJ, et al. Announcing cIMPACT-NOW: the consortium to inform molecular and practical approaches to CNS tumor taxonomy. Acta Neuropathol 2017;133:1-3.

19. Brat DJ, Aldape K, Colman H, et al. cIMPACT-NOW update 3: recommended diagnostic criteria for "Diffuse astrocytic glioma, IDH-wildtype, with molecular features of glioblastoma, WHO grade IV". Acta Neuropathol 2018;136:805-10. 